\title{
Interpretation of calcite growth data using the two-step crystal growth model
}

\author{
Clifford Y. Tai*, Meng-Chun Chang, Chi-Kao Wu, Yen-Chih Lin \\ Department of Chemical Engineering, National Taiwan University, Taipei 106, Taiwan
}

Received 15 June 2005; received in revised form 26 January 2006; accepted 25 March 2006

Available online 30 March 2006

\begin{abstract}
The crystal growth rates of calcite were evaluated in a dense fluidized-bed crystallizer using a constant-composition method. Several operation variables related to solution properties that affected growth rate, including supersaturation, $\mathrm{pH}$, ionic strength and activity ratio, were systematically investigated. Then the crystal growth-rate data were analyzed by the two-step crystal growth model and thus the mass-transfer and surface-reaction coefficients were obtained. The effects of the solution properties on the two individual coefficients were observed. Good explanations on these effects are needed.
\end{abstract}

(C) 2006 Elsevier Ltd. All rights reserved.

Keywords: Crystallization; Mass transfer; Surface reaction; Fluidization; Unit operations; Two-step growth model

\section{Introduction}

One of the water softening process is to feed a pellet reactor, which is a reactive, fluidized-bed, growth-type crystallizer, with drinking water containing calcium ions and a stream of carbonate aqueous solution (Dirken et al., 1990). Then calcium ions react with carbonate ions to form calcium carbonate and to grow on the seeds suspending in the crystallizer. The seeds are later removed from the reactor after they exceed a certain size. At this stage some fresh seeds are added to the crystallizer, and the crystallizer is operated in a semi-batch mode. In the design of a pellet reactor for water softening, the crystal growth kinetics of calcium carbonate is required.

In 1991 van Dijk and Wilms proposed an empirical model to express the calcite growth rate,

$$
\frac{\mathrm{d}\left[\mathrm{Ca}^{2+}\right]}{\mathrm{d} t}=K_{T} a\left\{\left[\mathrm{Ca}^{2+}\right]\left[\mathrm{CO}_{3}^{2-}\right]-K_{s p}^{\prime}\right\}
$$

where $\left(\mathrm{d}\left[\mathrm{Ca}^{2+}\right] / \mathrm{d} t\right)$ is the depletion rate of $\left[\mathrm{Ca}^{2+}\right]$ in the solution, which is equivalent to the calcite growth rate in moles

\footnotetext{
* Corresponding author. Tel./fax: +886223620832.

E-mail address: cytai@ntu.edu.tw (C.Y. Tai).
}

per unit time; $K_{T}$ is a constant; $a$ is the specific surface area of seed crystals; $K_{s p}^{\prime}$ is the concentration solubility product of calcite; and $\left\{\left[\mathrm{Ca}^{2+}\right]\left[\mathrm{CO}_{3}^{2-}\right]-K_{s p}^{\prime}\right\}$ represents the driving force for crystal growth. Eq. (1) is considered as an over-all growth rate model, because the driving force used is the difference between the concentration product of bulk solution and the solubility product of calcite. Tai et al. (1999) have demonstrated that the applicability of an over-all growth rate is confined to a limited range of operating variables when the two-step growth model is applied, which can be expressed mathematically by the following equations:

$G=K_{d}\left(\sigma-\sigma_{i}\right)$ diffusion,

$G=K_{r} \sigma_{i}^{r} \quad$ surface reaction.

Eq. (2) deals with the diffusion rate of solute between the bulk solution and the solution/crystal interface and Eq. (3) takes into account all the steps occurring on the crystal surface, including adsorption, desolvation, surface diffusion and incorporation, as proposed by Mullin (1993). Although this model is a simplified scheme for crystal growth, it reveals lots of useful kinetic information, which was summarized by Tai (1997).

The surface-reaction order of calcite is approximately 2 from different sources (Tai et al., 1993). This is also true for other systems, such as $\mathrm{CuSO}_{4} \cdot 5 \mathrm{H}_{2} \mathrm{O}$ (Tai and Pan, 1985) and potassium alum (Tai et al., 1987). Taking $r=2$, Eqs. (2) and (3) 
are combined to give

$$
\frac{\sigma}{\sqrt{G}}=\frac{\sqrt{G}}{K_{d}}+\frac{1}{\sqrt{K_{r}}}
$$

Thus the mass-transfer coefficient, $K_{d}$, and surface-reaction coefficient, $K_{r}$, can be evaluated from the slope and interception, respectively, of a plot, $(\sigma / \sqrt{G})$ vs. $\sqrt{G}$. In a well-suspended crystallizer, the mass-transfer coefficient is a function of system properties only, and can be evaluated by the following equation (Tai et al., 1987),

$S h\left(\frac{K_{d} L}{D}\right)=0.306 G a^{1 / 3} M v^{1 / 3} S c^{1 / 3}$.

Tai et al. (1999) studied the growth kinetics of calcite crystals suspended in a fluidized-bed crystallizer, which was maintained at a constant $\mathrm{pH}$, i.e., a pH-stat technique. They found that supersaturation, $\mathrm{pH}$ and ionic strength were significant variables to affect the growth rate of calcite crystals. In the same study a growth rate equation, which is based on the two-step growth model was proposed for design purpose,

$G=K_{d}\left[\left(1+\frac{K_{d}}{2 K_{r} \sigma}\right)-\sqrt{\left(1+\frac{K_{d}}{2 K_{r} \sigma}\right)^{2}-1}\right] \sigma$.

The disadvantage of the $\mathrm{pH}$-stat technique is that the supersaturation changes during an experiment and the crystal growth rate is determined from the slope of the calcium concentration profile in the crystallizer. Then the concentrations are converted to supersaturations. The crystal growth rate so obtained may be less accurate than that evaluated by the constantcomposition method, in which the supersaturation is kept constant.

Tai et al. (2005) studied the effects of solution properties, including supersaturation, $\mathrm{pH}$, activity ratio and ionic strength on the crystal growth rate of calcite in a fluidized-bed crystallizer using the constant-composition method. The growth experiments were performed in the metastable region, which was identified experimentally by Tai et al. (1999) using cured natural calcite in a size range between 390 and $921 \mu \mathrm{m}$. The crystal growth rates were evaluated from the volume depletion rate of titration solution. The results were consistent with those obtained by the $\mathrm{pH}$-stat method (Tai et al., 1999). However, the crystal growth-rate data of calcite were not further analyzed to explore the possible effects of solution properties on the different steps of crystal-growth process. Tai et al. (1999) applied the two-step crystal growth model to investigate the effects of particle size and superficial velocity on the mass-transfer coefficient and surface-reaction coefficient of calcite growth in a dense fluidized bed. They found that the mass-transfer coefficient was independent of crystal size and superficial velocity and the surface-reaction coefficient is size dependent but independent of superficial velocity. These results are similar to that of readily soluble systems, such as potassium alum (Budz et al., 1984; Tai et al., 1987) and nickel sulfate (Phillips and Epstein, 1974). Besides, the two-step crystal model has been employed to analyze the crystal growth rate data of many readily soluble systems and much useful kinetic information has been revealed (Tai, 1997). It is interesting to see how the solution properties will influence the mass-transfer and surfacereaction coefficient.

The aim of this study is to investigate the crystal growth kinetics of calcite in a dense fluidized-bed crystallizer using the constant-composition method. Because there are too many factors that affect crystal growth rate of sparingly soluble system, the variables investigated in this study are confined to solution properties. The growth experiments were performed in the metastable region by keeping the same crystal size, superficial velocity and temperature. The calcite crystal growth rates were measured at various supersaturations under specified $\mathrm{pH}$, activity ratio, and ionic strength. The crystal growth rate of calcite was evaluated from the volume depletion rate of titration solution. The crystal growth rate data were further analyzed by the two-step crystal growth model in order to explore the effect of the solution properties on the masstransfer coefficient and surface-reaction coefficient, which were evaluated by Eq. (4). Finally, a possible controlling step of calcite growth process was postulated for various operation conditions.

\section{Experimental}

The experimental apparatus and procedures have been reported somewhere else (Tai et al., 2005) and will be described briefly here. A voltage regulator was installed to the experimental system in order to reduce noise caused by unstable voltage. The crystallization system shown in Fig. 1 contains a fluidized bed, which is a PVC column with a distributor at the bottom to give an even flow and with an enlarged section on the top to prevent the seed crystals from carrying over to the storage tank, a storage tank for holding solution, and a composition control system, which consists of two autotitrators for adding reaction solutions and $\mathrm{NaOH}$ solution to keep the system at constant composition and $\mathrm{pH}$. Usually the amount of $\mathrm{NaOH}$ added to the system was only a few drops. During an experimental run, the chloride and sodium ion would accumulate to increase the ionic strength of solution due to a semibatch operation. However, the change in growth rate was negligible due to an increment of ionic strength within $5 \%$. The volume of titration solution added to the system was recorded automatically on the autotitrator after the seed crystals were charged into the crystallizer and the solution composition and $\mathrm{pH}$ became steady. The titration curve was used to calculate the crystal growth rate. After an experimental run, which usually lasted for one hour, the seed crystals were removed, washed and then kept in a saturated solution for next experiment. During the experiment, the seed size, superficial velocity, and temperature were kept at $774 \mu \mathrm{m}, 4.7 \times 10^{-2} \mathrm{~m} / \mathrm{s}$ and $298 \mathrm{~K}$, respectively. 


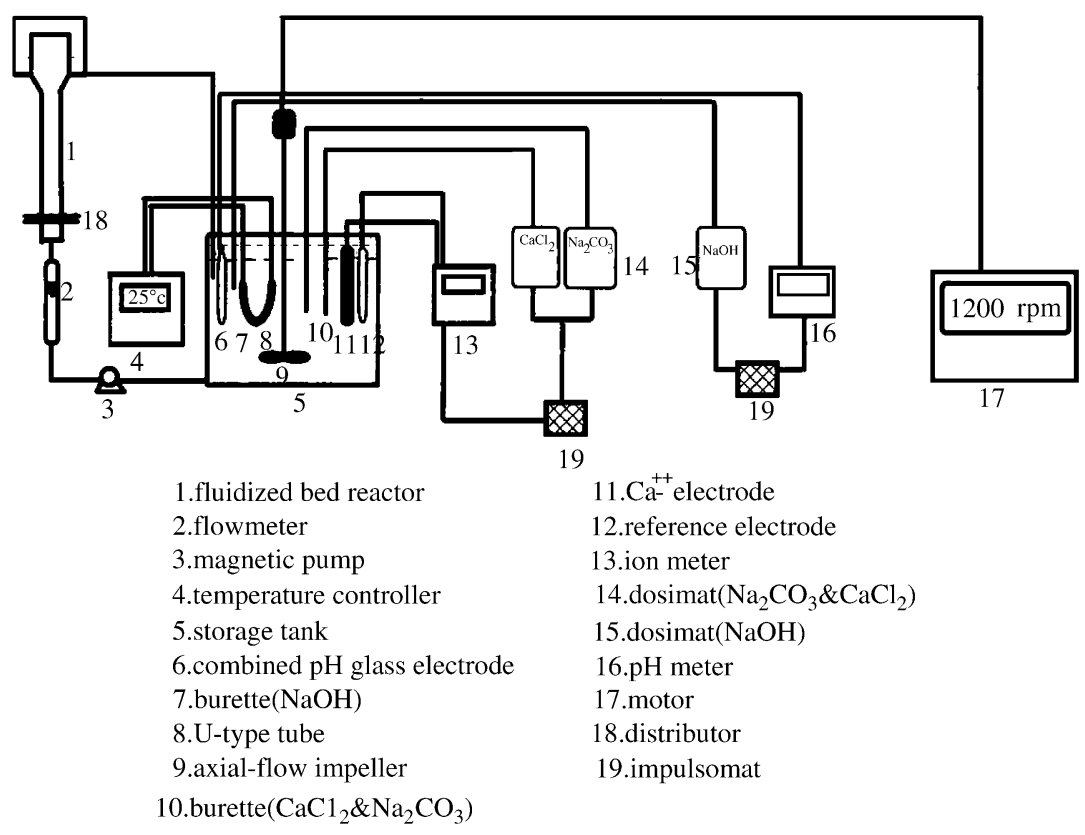

Fig. 1. A constant-composition crystallization system.

\section{Determination of crystal growth rate}

The crystal growth rate in $\mathrm{kg} / \mathrm{m}^{2} \mathrm{~s}$ is expressed as

$R_{g}=\frac{1}{A} \frac{\mathrm{d} W}{\mathrm{~d} t}$,

where $W$ and $A$ are the total mass and surface area of crystals, which are related to the crystal size by the following forms:

$W=n \rho_{p} \alpha L^{3}$,

$A=n \beta L^{2}$,

where $\rho_{p}$ is the crystal density; $\alpha$ and $\beta$ are the volume and the surface area shape factors, respectively; and $n$ is the number of crystals.

Substituting Eqs. (8) and (9) into Eq. (7) gives

$R_{g}=\frac{3 \alpha \rho_{p}}{\beta} \frac{\mathrm{d} L}{\mathrm{~d} t}=\frac{3}{L} \frac{W}{A} \frac{\mathrm{d} L}{\mathrm{~d} t}$.

Then Eqs. (7) and (10) can be combined to give the linear crystal growth rate in $\mathrm{m} / \mathrm{s}$,

$G=\frac{\mathrm{d} L}{\mathrm{~d} t}=\frac{L}{3 W} \frac{\mathrm{d} W}{\mathrm{~d} t}$.

In a constant-composition operation, the solution volume increased but the concentration kept constant. To evaluate $\mathrm{d} W / \mathrm{d} t$, the change in volume should be taken into consideration. Thus,

$$
\begin{aligned}
\frac{\mathrm{d} W}{\mathrm{~d} t} & =M \cdot \frac{\mathrm{d}\left\{V_{o}\left[\mathrm{Ca}^{2+}\right]_{o}+V_{a}\left[\mathrm{Ca}^{2+}\right]_{a}-\left(V_{o}+V_{a}\right)\left[\mathrm{Ca}^{2+}\right]_{o}\right\}}{\mathrm{d} t} \\
& =M \cdot\left(\left[\mathrm{Ca}^{2+}\right]_{a}-\left[\mathrm{Ca}^{2+}\right]_{o}\right) \frac{\mathrm{d} V_{a}}{\mathrm{~d} t}
\end{aligned}
$$

where $M$ is the molecular weight of $\mathrm{CaCO}_{3}, V_{o}$ and $V_{a}$ are the volume of original solution and added solution, respectively, and $\left[\mathrm{Ca}^{2+}\right]_{o}$ and $\left[\mathrm{Ca}^{2+}\right]_{a}$ represent the $\mathrm{Ca}^{2+}$ concentration of the original solution and added solution, respectively.

Eq. (12) is substitute into Eq. (11) to yield

$G=\frac{L M}{3 W}\left(\left[\mathrm{Ca}^{2+}\right]_{a}-\left[\mathrm{Ca}^{2+}\right]_{o}\right) \frac{\mathrm{d} V_{a}}{\mathrm{~d} t}$.

The volume of titration solution was recorded automatically as shown in Fig. 2. Then the titration curve was fitted by a straight line. The slope of the straight line was used to calculate the crystal growth rate according to Eq. (13). It should be noted that the growth rate determined in this study is the average linear growth rate of all crystal faces. Since the crystal growth rate of $\mathrm{CaCO}_{3}$ was very slow, in the order of $10^{-10} \mathrm{~m} / \mathrm{s}$, it was evaluated by the depletion of titration volume, then converted to linear growth rate. Therefore the two-step growth model described in this paper is based on 1-D analysis.

Once the crystal growth rates are available at different supersaturations, the mass-transfer coefficient, $K_{d}$, and the surfacereaction coefficient, $K_{r}$, can be evaluated by Eq. (4), in which the relative supersaturation, $\sigma$, was defined by Nielsen and Toft (1984).

$\sigma=\left(\frac{K_{i p}}{K_{s p}}\right)^{1 / 2}-1$

A computer program, which contains mass-action equation, mass-balance equation, charge-balance equation, and the modified Debye-Hückel equation for estimating the activity coefficient, was used to calculate the relative supersaturation (Nancollas, 1966; Tai et al., 1993). 


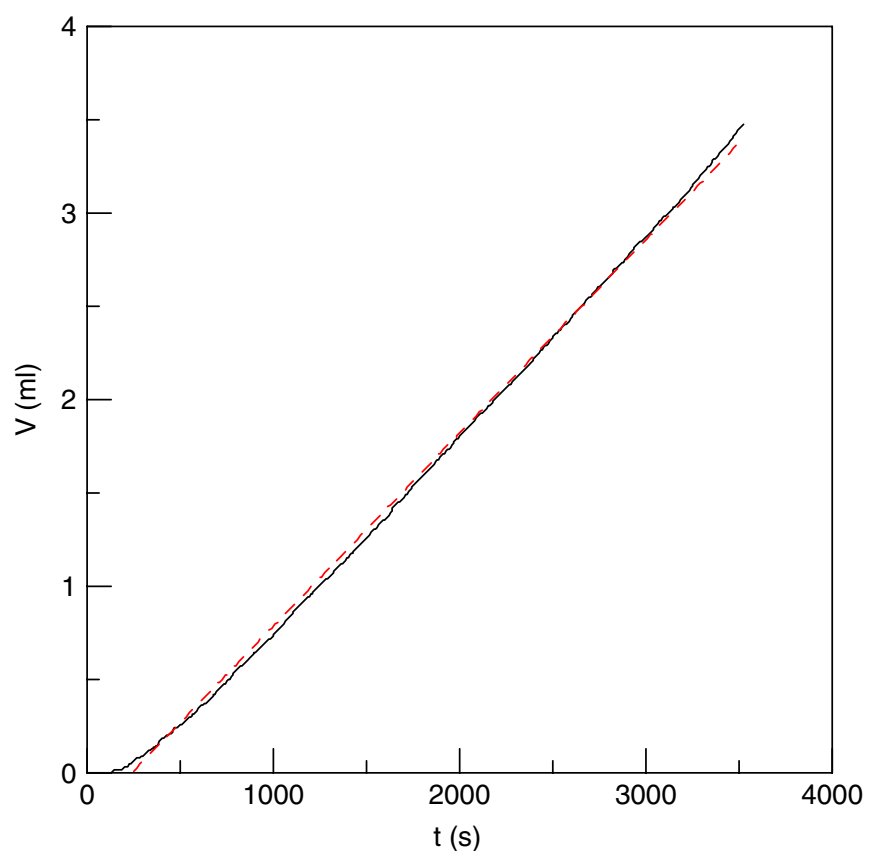

Fig. 2. A titration curve at the following operation conditions: $\sigma=1, \mathrm{pH}=9.5$, $R=4.0$, and $I=0.018 \mathrm{M}$. - experimental line; - - - - fitted line.

On the other hand, the crystal growth rate of calcite in a $\mathrm{pH}$-stat operation was estimated by the following equation (Tai et al., 1999):

$G=\frac{L M V_{o}}{3 W}\left(-\frac{\mathrm{d}\left[\mathrm{Ca}^{2+}\right]}{\mathrm{d} t}\right)$.

The concentration profile of calcium ion for the samples withdrawn intermittently from the crystallizer was determined by an atomic absorption spectrometer. Then the concentration profile was fitted by a polynomial, which could be converted to crystal growth rate by taking derivative first and then by using Eq. (15). It is clear that the two techniques for measuring crystal growth rate are quite different by comparing Eqs. (13) and (15).

\section{Results and discussion}

\subsection{Assurance of constant-composition operation}

In this experiment the fluidized-bed crystallizer was operated in a dense mode, i.e., a clear boundary existing between solution and suspension for the superficial velocity being operated below the terminal velocity of particles. A dense bed was chosen instead of a lean bed, which is operated at a superficial velocity near the terminal velocity of particles, because it is more advantageous as far as seed loading is concerned. Suppose a lean bed was used, the operation time should last longer due to a slow growth rate of calcite.

If the solution composition in the system was controlled by a $\mathrm{pH}$-electrode, a constant composition was difficult to maintain as shown in Fig. 3. A possible reason was that the absorption of $\mathrm{CO}_{2}$ from the environment caused a change in $\mathrm{pH}$ that triggered the autotitrator. Therefore the calcium ion increased

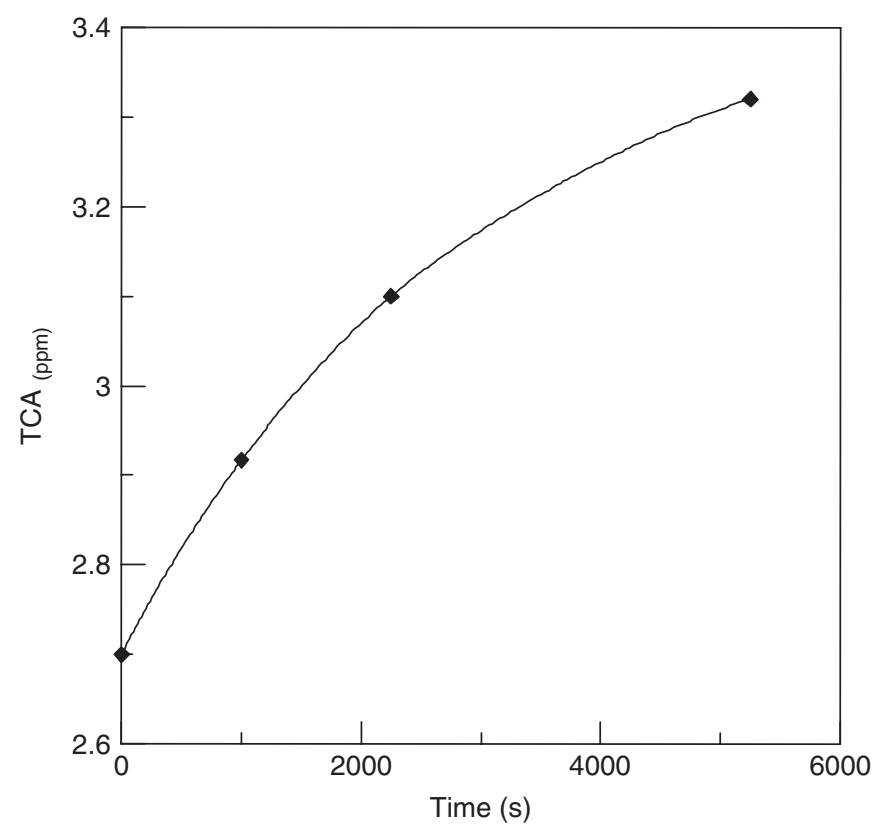

Fig. 3. Total calcium concentration profile using a combined pH-glass electrodes to maintain constant composition.

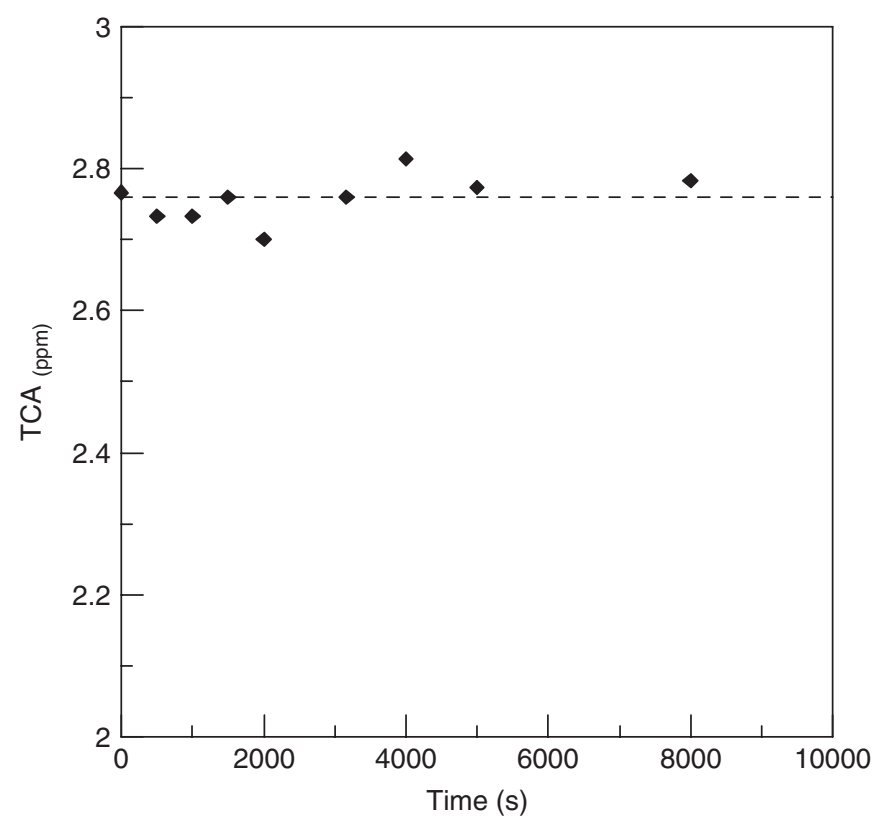

Fig. 4. Total calcium concentration profile using a calcium ion-selective electrode to maintain constant composition.

gradually. To overcome the problem, two sets of autotitrators, one equipped with a calcium-ion electrode and the other one with a $\mathrm{pH}$ electrode, were applied to control the added amount of reaction solutions and $\mathrm{NaOH}$ solution, respectively.

To check the constant composition of crystallization solution, solution samples were withdrawn intermittently by using a syringe fitted with a $0.22 \mu \mathrm{m}$ filter, and the calcium ion concentration was determined by an atomic absorption spectrometer. The results shown in Fig. 4, for example, indicate that the 


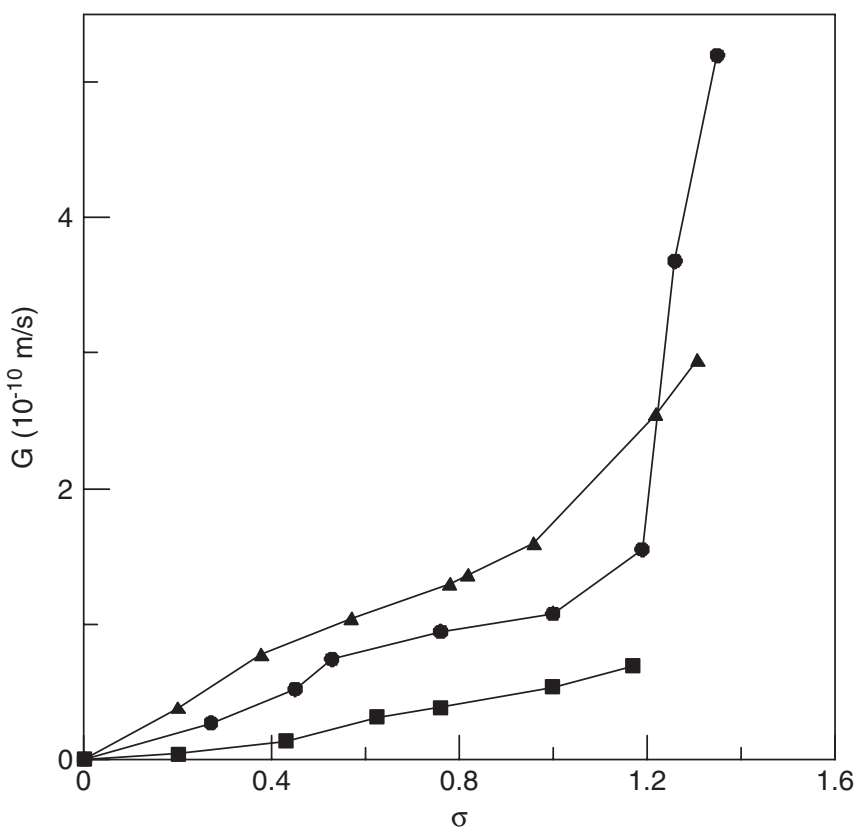

Fig. 5. Crystal growth rate of calcite as a function of $\sigma$ for three levels of pH: $8.5(\square), 9.0(\mathbf{\square})$, and $9.5(\mathbf{\Delta})$. Other operation variables kept constant are: $R=5.5$ and $I=0.018 \mathrm{M}$.

calcium concentration is nearly constant during a long period of operation up to $8000 \mathrm{~s}$, although the calcium concentration fluctuates a little bit around the initial value.

\subsection{Effects of $p H$ on $K_{d}$ and $K_{r}$}

The $\mathrm{pH}$ range investigated in this study was between 8.5 and 9.5, which is about the range used in a pellet reactor for water softening (Van Dijk and Wilms, 1991). It will encounter difficulties beyond this $\mathrm{pH}$ range in the operation for kinetic study. If the $\mathrm{pH}$ value is higher than 9.5 , carbon dioxide will be absorbed from air to mess up the measurement of growth rate. On the other hand, the growth rate will be very slow at a $\mathrm{pH}$ below 8 because the concentration of $\mathrm{CO}_{3}^{2-}$ is too low (ASTM, 1974).

The calcite growth rates at various relative supersaturation, $\sigma$ from 0.20 to 1.35 , were measured for three $\mathrm{pH}$ levels, i.e., 8.5, 9.0 and 9.5 , with other operation variables kept the same. The growth rate data are plotted in Fig. 5. General trends observed are that the growth rate increases with an increase in supersaturation and $\mathrm{pH}$, and the growth rates become very large when $\sigma$ is above 1.2. A large increase in growth rate for $\sigma$ above 12 was due to a sudden increase of diffusivity and will be further explained later.

When the growth rate data were analyzed by the two-step model of crystal growth by taking $r=2$ (Tai et al., 1993), a plot of Eq. (4) was constructed as shown in Fig. 6 for three levels of $\mathrm{pH}$. For each set of data, a straight line for the first six data points follows Eq. (4). However, the straight line for $\mathrm{pH}=9.5$ has an intercept of negative value, which results in a negative $K_{r}$ with no physical meaning. Thus the growth-rate data for $\mathrm{pH}=9.5$ shown in Fig. 5 were fitted by a straight line

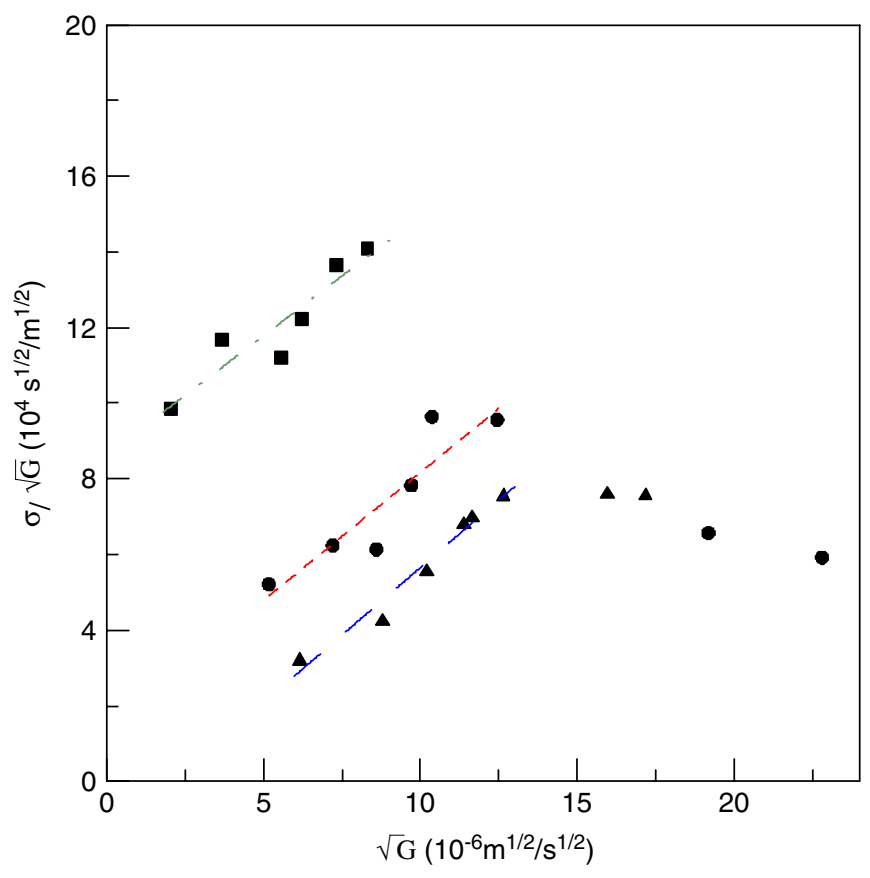

Fig. 6. Calcite growth-rate data plotted by the two-step growth model with $r=2$ at three $\mathrm{pH}$ values: $8.5(\boldsymbol{\square}), 9.0(\mathbf{O})$, and $9.5(\boldsymbol{\Delta})$.

Table 1

Growth kinetics of calcite at various $\mathrm{pH}$ levels

\begin{tabular}{lllll}
\hline $\mathrm{pH}$ value & Fitted equation & $\begin{array}{l}\text { Correlation } \\
\text { coefficient }\end{array}$ & $\begin{array}{l}K_{d} \\
(\mathrm{~m} / \mathrm{s})\end{array}$ & $\begin{array}{l}K_{r} \\
(\mathrm{~m} / \mathrm{s})\end{array}$ \\
\hline $9.5 \quad Y^{\mathrm{a}}=1.52 X^{\mathrm{b}}$ & 0.98 & 1.52 & - \\
9.0 & $Y^{\mathrm{c}}=0.675 X^{\mathrm{d}}$ & 0.83 & 1.48 & 50.3 \\
8.5 & $Y^{\mathrm{c}}=0.630 X^{\mathrm{d}}+8.627$ & 0.86 & 1.58 & 1.34 \\
\hline${ }^{\mathrm{a}} Y=G \mathrm{~m} / \mathrm{s}$. & & & \\
${ }^{\mathrm{b}} X=\sigma$. & & & \\
${ }^{\mathrm{c}} Y=(\sigma / \sqrt{G}) \times 10^{4} \mathrm{~s}^{1 / 2} / \mathrm{m}^{1 / 2}$. & & & \\
${ }^{\mathrm{d}} X=\sqrt{G} \times 10^{-6} \mathrm{~m}^{1 / 2} / \mathrm{s}^{1 / 2}$. & & &
\end{tabular}

with a correlation coefficient of 0.98 and the intercept almost pass the origin. It means that the calcite growth at $\mathrm{pH}=9.5$ is diffusion-control. The calculated $K_{d}$ from Fig. 6 is $1.58 \times$ $10^{-10}$ and $1.48 \times 10^{-10} \mathrm{~m} / \mathrm{s}$ for $\mathrm{pH}=8.5$ and 9.0 , respectively, and from Fig. 5 for $\mathrm{pH}=9.5$ is $1.52 \times 10^{-10} \mathrm{~m} / \mathrm{s}$. The fitted equations and their associated correlation coefficient and the calculated individual coefficients are tabulated in Table 1. The largest difference is less than $10 \%$ between $K_{d}$ 's. It implies that $\mathrm{pH}$ has no influence on the diffusion step. On the other hand, $K_{r}$ increases by an order, i.e., from $1.34 \times 10^{-10}$ to $5.03 \times 10^{-9} \mathrm{~m} / \mathrm{s}$, for $\mathrm{pH}$ changing from 8.5 to 9.0 , and the change in $K_{r}$ is even higher when $\mathrm{pH}$ increases from 9.0 to 9.5 . The results are not surprising because surface reaction takes place at solution/crystal interface and the interface conditions change with $\mathrm{pH}$ value. In the region of higher growth rate or higher supersaturation, the growth-rate data do not fit Eq. (4) as shown in Fig. 6 for $\mathrm{pH}=8.5$ and 9.0, and do not follow straight line shown in Fig. 5 for $\mathrm{pH}=9.5$ either. A similar phenomenon was observed for the growth of potassium alum (Tai et al., 


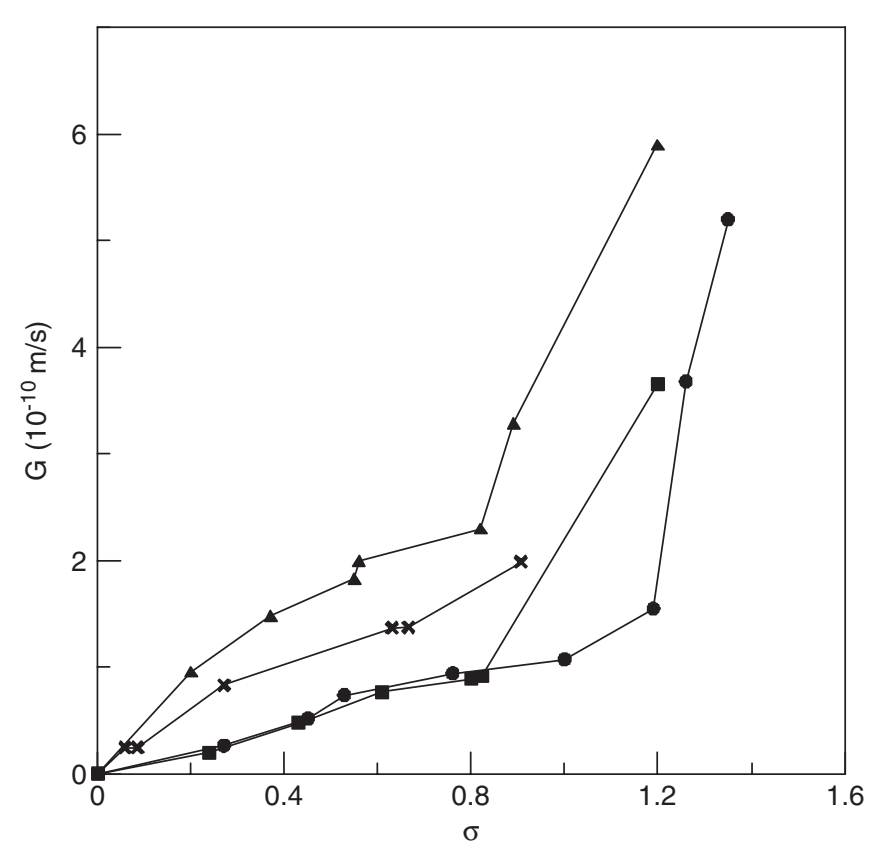

Fig. 7. Calcite growth rates as a function of $\sigma$ for four levels of $R: 5.5(\bigcirc)$, $2.0(\times), 1.0(\mathbf{\Delta})$, and $0.4(\mathbf{\square})$. Other operation variables kept constant are: $\mathrm{pH}=9$ and $I=0.018 \mathrm{M}$.

1987) and nickel sulfate (Phillips and Epstein, 1974) grown in a fluidized bed. The data points were lower than that predicted by Eq. (4). It is attributed to an increase in $K_{d}$, which increases with diffusivity, at higher supersaturation. For potassium alum system Fukui and Nakajima (1985) measured diffusivity using the interferometric method. They reported that the diffusivity remained quite constant up to a supersaturation $(\sigma=0.95)$, then it started to increase appreciably.

The effect of $\mathrm{pH}$ on other sparingly soluble salt has been reported by Stubicar et al. (1993). They found that the growth rate of lead fluoride had a maximum at its isoelectric point, which was at $\mathrm{pH}=5.6$. The isoelectric point of calcium carbonate is between 9 and 10 (Reed, 1989). The growth rate of calcite, which is one form of calcium carbonate, increases with $\mathrm{pH}$ in the range of 8.5 and 9.5. This is consistent qualitatively with the result reported by Stubicar et al. (1993) for lead fluoride.

\subsection{Effect of activity ratio on $K_{d}$ and $K_{r}$}

The activity ratio of calcium carbonate is defined as: $R=$ $a_{\mathrm{Ca}^{2+}} / a_{\mathrm{CO}_{3}^{2-}}=\left[\mathrm{Ca}^{2+}\right] \cdot y_{\mathrm{Ca}^{2+}} /\left[\mathrm{CO}_{3}^{2-}\right] \cdot y_{\mathrm{CO}_{3}^{2-}}$. In the estimation of activity, $y_{i}$, for $\mathrm{Ca}^{2+}$ and $\mathrm{CO}_{3}^{2-}$, the activity coefficient was calculated by the modified Debye-Hückel equation proposed by Davies (Butler, 1964). Tai et al. (2005) studied the effect of activity ratio on the crystal growth rate of $\mathrm{CaCO}_{3}$ by keeping other operation variables constant, including $\mathrm{pH}$, ionic strength, supersaturation, seed size and superficial velocity. They found that there existed a maximum growth rate around $R=2.5$. Therefore, it is interesting to know how the activity ratio affects $K_{d}$ and $K_{r}$. The growth-rate data of calcite crystal are plotted in Fig. 7 for $R=0.4,1.0,2.0$, and 5.5. Similar to the results

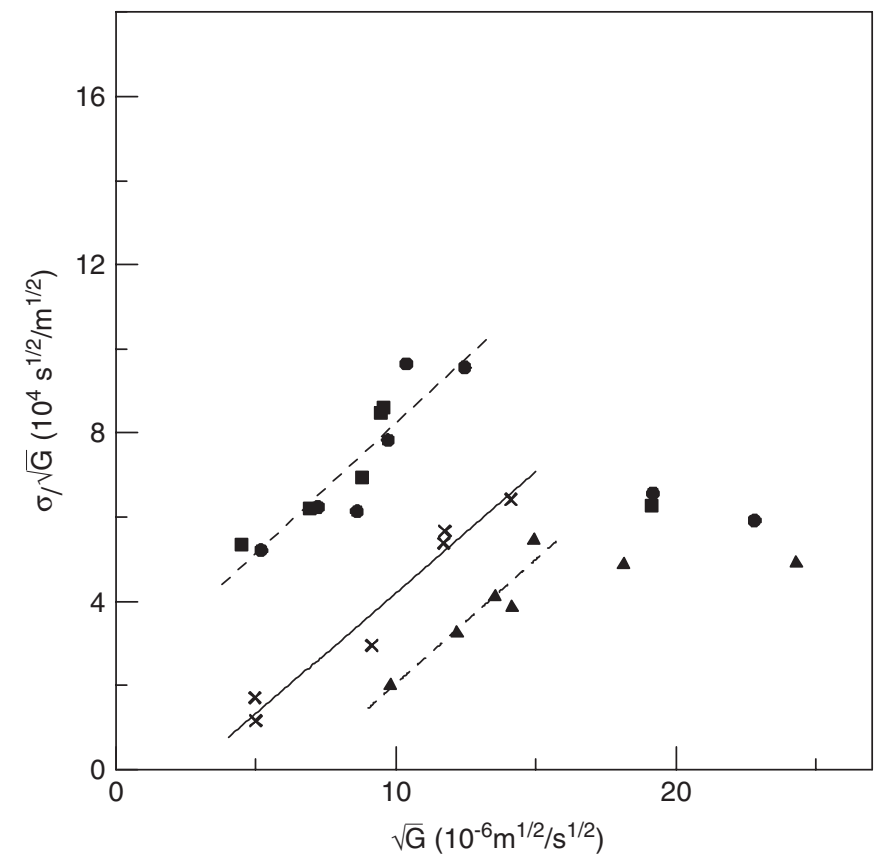

Fig. 8. Calcite growth-rate data plotted by the two-step growth model with $r=2$ at three ionic-activity ratios: $5.5(\mathbf{O}), 2.0(\times), 1.0(\mathbf{\Delta})$, and $0.4(\mathbf{\square})$. Other variables kept constant are: $I=0.018 \mathrm{M}$ and $\mathrm{pH}=9$.

reported by Tai et al. (2005), a maximum was found for the growth rate, which has the highest value at $R=1$ instead of 2.5 reported by Tai et al. (2005). However, the operation conditions are not identical for the two cases, only ionic strength kept the same. Besides, the growth-rate data for $R=0.4$ almost mingle with that for $R=5.5$. Then the growth-rate data are rearranged and plotted as $\sigma / \sqrt{G}$ versus $\sqrt{G}$ shown in Fig. 8. The growthrate data of $R=0.4$ and 5.5 were fitted with one straight line in the linear regression analysis because the growth-rate data are difficult to separate. Similar to Fig. 6, straight lines were obtained at lower growth rates and data points at higher growth rate deviated from the straight lines. The intercept of the line for $R=1$ and 2 were negative, thus the growth rate data of $R=1$ and 2 shown in Fig. 7 were fitted by straight lines. The fitted equations and their associated correlationcoefficients and the slopes of the fitted straight lines, i.e., $K_{d}$, are tabulated in Table 2, in which the fitted straight line shown in Fig. 8 for the growth rate data of $R=0.4$ and 5.5 is also presented. From Table 2 we know that $K_{r}$ for $R=1$ and 2 is too large to determine and the largest $K_{d}$ is about two-fold of the smallest $K_{d}$. It is concluded that the crystal growth process of calcite at $R=1$ or 2 is diffusion controlled, and the activity ratio has a greater influence on the surface-reaction step. Stubicar et al. (1990) investigated the effect of $\mathrm{Pb} / \mathrm{F}$ activity ratio on the growth kinetics of lead fluoride. They found that a plot of growth rate versus supersaturation gave different slopes, 1.55 for $a_{\mathrm{Pb}^{2+}} / a_{\mathrm{F}^{-}}>1$ and 4.00 for $a_{\mathrm{Pb}^{2+}} / a_{\mathrm{F}^{-}}<1$. Although the crystal growth kinetics of $\mathrm{CaCO}_{3}$ and $\mathrm{PbF}_{2}$ respond differently to different activity ratios, the effects of activity ratio on the sparingly soluble salts are significant. 
Table 2

Growth kinetics of calcite at various values of activity ratio

\begin{tabular}{|c|c|c|c|c|}
\hline $\begin{array}{l}\text { Activity ratio } \\
(R)\end{array}$ & Fitted equation & $\begin{array}{l}\text { Correlation } \\
\text { coefficient }\end{array}$ & $\begin{array}{l}K_{d} \\
(\mathrm{~m} / \mathrm{s})\end{array}$ & $\begin{array}{l}K_{r} \\
(\mathrm{~m} / \mathrm{s})\end{array}$ \\
\hline 1.0 & $Y^{\mathrm{a}}=2.86 X^{\mathrm{b}}+0.360$ & 0.90 & $2.86 \times 10^{-10}$ & - \\
\hline 2.0 & $Y^{\mathrm{a}}=1.98 X^{\mathrm{b}}+0.146$ & 0.98 & $1.98 \times 10^{-10}$ & - \\
\hline 0.4 and 5.5 & $Y^{\mathrm{c}}=0.63 X^{\mathrm{d}}+1.959$ & 0.83 & $1.58 \times 10^{-10}$ & $2.61 \times 10^{-9}$ \\
\hline
\end{tabular}

${ }^{\mathrm{a}} Y=G \mathrm{~m} / \mathrm{s}$

${ }^{\mathrm{b}} X=\sigma$

${ }^{\mathrm{c}} Y=(\sigma / \sqrt{G}) \times 10^{4} \mathrm{~s}^{1 / 2} / \mathrm{m}^{1 / 2}$

${ }^{\mathrm{d}} X=\sqrt{G} \times 10^{-6} \mathrm{~m}^{1 / 2} / \mathrm{s}^{1 / 2}$

\subsection{Effect of ionic strength on $K_{d}$ and $K_{r}$}

The ionic strength of a solution is calculated by the following equation:

$I=\frac{1}{2} \sum_{i=1}^{n} Z_{i}^{2} C_{i}$,

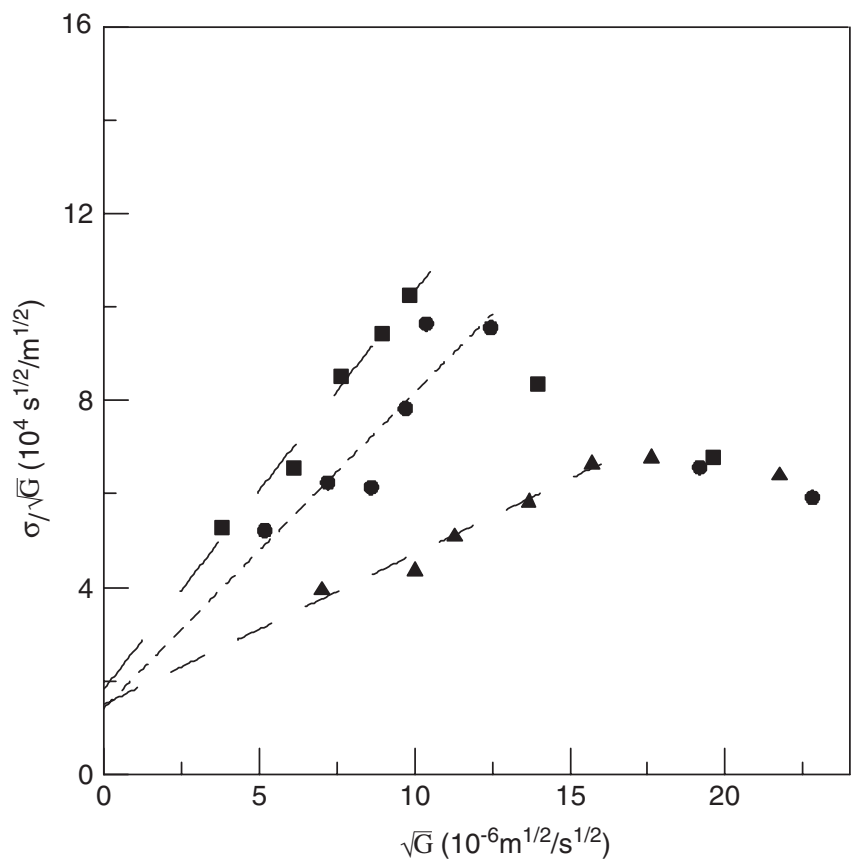

Fig. 9. Calcite growth-rate data plotted by the two-step growth model with $r=2$ at three levels of ionic strength: $0.005 \mathrm{M}(\square), 0.018 \mathrm{M}(\mathbf{\square})$, and $0.035 \mathrm{M}$

$(\boldsymbol{\Delta})$. Other variables kept constant are: $\mathrm{pH}=9.0$ and $R=5.5$. where $Z_{i}$ is the valence of $i$ species and $C_{i}$ includes the concentration of $\mathrm{H}^{+}, \mathrm{OH}^{-}, \mathrm{Ca}^{2+}, \mathrm{CaOH}^{+}, \mathrm{CaHCO}_{3}^{+}, \mathrm{HCO}_{3}^{-}, \mathrm{CO}_{3}^{2-}$, $\mathrm{Na}^{+}$and $\mathrm{Cl}^{-}$(Tai et al., 1993). In this experiment, the ionic strength was adjusted by adding $\mathrm{NaCl}$ to the solution because $\mathrm{Na}^{+}$and $\mathrm{Cl}^{-}$were already present in the solution.

Stubicar et al. (1993) reported that the linear growth rate of lead fluoride in a high ionic strength solution $\left(0.1 \mathrm{~mol} / \mathrm{dm}^{3}\right.$ $\mathrm{KNO}_{3}$ solution), i.e., at conditions of particle charge compensation, is almost five times greater than that in a low ionic strength solution, which is an aqueous solution without adding $\mathrm{KNO}_{3}$. The difference is tremendous so that a further study on the ionic strength to reveal the growth mechanism is worthy.

The crystal growth rates at various supersaturations, from $\sigma=0.2$ to 1.4 , were measured for three levels of ionic strength, i.e., $0.005,0.018$ and $0.035 \mathrm{M}$, with other operation variables kept the same. The growth rate increases with an increase in ionic strength and supersaturation. Under the same supersaturation, a big difference by a factor of 3 in growth rate was found between the highest and the lowest level of ionic strength, a result somewhat similar to that reported by Stubicar et al. (1993). According to Eq. (4), the growth-rate data were rearranged to plot $\sigma / \sqrt{G}$ versus $\sqrt{G}$ as shown in Fig. 9. It is found that the straight lines tend to converge on the axis of ordinate. According to Eq. (4) the calculated $K_{d}$ 's and $K_{r}$ 's are tabulated in Table 3, in which the fitted equations and their associated correlation coefficients are also listed. Judging from the correlation coefficients, which are $0.98,0.83$ and 0.96 , the fitting of straight lines is acceptable. The value of $K_{d}$ varies from $1.17 \times 10^{-10}$ to $3.11 \times 10^{-10} \mathrm{~m} / \mathrm{s}$, thus the ionic strength has a great influence on $K_{d}$. On the other hand, $K_{r}$ is less affected by ionic strength, changing from $3.03 \times 10^{-9}$ to $6.40 \times 10^{-9} \mathrm{~m} / \mathrm{s}$. Besides, $K_{r}$ is greater than $K_{d}$ by an order, meaning that the surface-reaction resistance is less significant than the

Table 3

Growth kinetics of calcite at various levels of ionic strength

\begin{tabular}{|c|c|c|c|c|}
\hline $\begin{array}{l}\text { Ionic strength } \\
\text { (M) }\end{array}$ & Fitted equation & $\begin{array}{l}\text { Correlation } \\
\text { coefficient }\end{array}$ & $\begin{array}{l}K_{d} \\
(\mathrm{~m} / \mathrm{s})\end{array}$ & $\begin{array}{l}K_{r} \\
(\mathrm{~m} / \mathrm{s})\end{array}$ \\
\hline 0.005 & $Y^{\mathrm{a}}=0.8519 X^{\mathrm{b}}+1.814$ & 0.98 & $1.17 \times 10^{-10}$ & $3.03 \times 10^{-9}$ \\
\hline 0.018 & $Y^{\mathrm{a}}=0.6673 X^{\mathrm{b}}+0.1251$ & 0.83 & $1.49 \times 10^{-10}$ & $6.40 \times 10^{-9}$ \\
\hline 0.035 & $Y^{\mathrm{a}}=0.3217 X^{\mathrm{b}}+1.495$ & 0.96 & $3.11 \times 10^{-10}$ & $4.47 \times 10^{-9}$ \\
\hline
\end{tabular}

${ }^{\mathrm{a}} Y=\sigma / \sqrt{G} \times 10^{4} \mathrm{~s}^{1 / 2} / \mathrm{m}^{1 / 2}$.

${ }^{\mathrm{b}} X=\sqrt{G} \times 10^{-6} \mathrm{~m}^{1 / 2} / \mathrm{s}^{1 / 2}$. 
mass-transfer resistance under the specified operation conditions for a second-order surface-reaction rate (Tai et al., 1993).

\section{Conclusion}

A fluidized-bed crystallizer controlled at constant composition was used to measure the crystal growth rate of calcite. Several operation variables were studied in this experiment, including supersaturation, $\mathrm{pH}$, activity ratio and ionic strength. These factors are significant in a range of relative supersaturation $(\sigma)$ between 0.2 and 1.4 , the $\mathrm{pH}$ value between 8.5 and 9.5, the ionic strength $(I)$ between 0.0025 and $0.0034 \mathrm{M}$, and the activity ratio $(R)$ between 0.4 and 5.5 . The crystal growth rate increases with an increase of supersaturation, $\mathrm{pH}$ value, and ionic strength, however, the growth rate shows a maximum at an activity ratio around 1 .

When the growth-rate data were analyzed by the two-step crystal growth model by assuming $r=2$, the calculated masstransfer coefficient is rather independent of $\mathrm{pH}$ and activity ratio, but varies with ionic strength, the surface-reaction coefficient increases with $\mathrm{pH}$ and activity ratio but rather independent of ionic strength. As far as the resistance of calcite growth process is concerned, the surface-reaction resistance is less significant in the range of the operation conditions investigated in this study.

\section{Notation}

\begin{tabular}{|c|c|}
\hline$a$ & specific surface area of crystals, $\mathrm{m}^{2} / \mathrm{kg}$ \\
\hline$A$ & surface area of crystals, $\mathrm{m}^{2}$ \\
\hline$C_{i}$ & concentration of $i$ species, $\mathrm{mol} / \mathrm{m}^{3}$ \\
\hline$\left[\mathrm{Ca}^{2+}\right]$ & calcium-ion concentration, $\mathrm{mol} / \mathrm{m}^{3}$ \\
\hline$\left[\mathrm{Ca}^{2+}\right]_{a}$ & $\begin{array}{l}\text { calcium-ion concentration of titration solution, } \\
\mathrm{mol} / \mathrm{m}^{3}\end{array}$ \\
\hline$\left[\mathrm{Ca}^{2+}\right]_{o}$ & $\begin{array}{l}\text { calcium-ion concentration of original solution, } \\
\mathrm{mol} / \mathrm{m}^{3}\end{array}$ \\
\hline$\left[\mathrm{CO}_{3}^{2-}\right]$ & carbonate-ion concentration, $\mathrm{mol} / \mathrm{m}^{3}$ \\
\hline$D$ & diffusivity of solute in solution, $\mathrm{m}^{2} / \mathrm{s}$ \\
\hline$G$ & linear crystal growth rate, $\mathrm{m} / \mathrm{s}$ \\
\hline Ga & Galileo number, dimensionless \\
\hline$I$ & ionic strength, $\mathrm{M}$ \\
\hline$K_{d}$ & mass-transfer coefficient, $\mathrm{m} / \mathrm{s}$ \\
\hline$K_{i p}$ & ionic product, $(\mathrm{mol})^{2} / \mathrm{m}^{6}$ \\
\hline$K_{r}$ & surface-reaction coefficient, $\mathrm{m} / \mathrm{s}$ \\
\hline$K_{s p}$ & solubility product, $(\mathrm{mol})^{2} / \mathrm{m}^{6}$ \\
\hline$K_{s p}^{\prime}$ & concentration solubility product, $(\mathrm{mol})^{2} / \mathrm{m}^{6}$ \\
\hline$K_{T}$ & proportional constant in Eq. (1) \\
\hline$L$ & crystal size, $\mathrm{m}$ \\
\hline$M$ & molecular weight of $\mathrm{CaCO}_{3}, \mathrm{~kg} / \mathrm{kmol}$ \\
\hline$M_{v}$ & density number, $\left[\left(\rho_{p}-\rho\right) / \rho\right]$, dimensionless \\
\hline$n$ & number of crystals, dimensionless \\
\hline$r$ & surface reaction order, dimensionless \\
\hline$R$ & activity ratio, dimensionless \\
\hline$R_{g}$ & crystal growth rate, $\mathrm{kg} / \mathrm{m}^{2} \mathrm{~s}$ \\
\hline$S c$ & Schmidt number, dimensionless \\
\hline$S h$ & Sherwood number, dimensionless \\
\hline
\end{tabular}

$t \quad$ time, $\mathrm{s}$

$V_{a} \quad$ titration volume, $\mathrm{m}^{3}$

$V_{o} \quad$ volume of original solution, $\mathrm{m}^{3}$

$u \quad$ superficial velocity, $\mathrm{m} / \mathrm{s}$

W crystal weight, $\mathrm{kg}$

$y_{\mathrm{Ca}^{2+}} \quad$ activity coefficient of calcium ion, dimensionless

$y_{\mathrm{CO}_{3}^{2-}} \quad$ activity coefficient of carbonate ion, dimensionless

$Z_{i} \quad$ valence of $i$ species, dimensionless

\section{Greek letters}

$\alpha \quad$ volume shape factor of crystal, dimensionless

$\beta \quad$ surface area shape factor of crystal, dimen-

sionless

$\rho \quad$ solution density, $\mathrm{kg} / \mathrm{m}^{3}$

$\rho_{p} \quad$ crystal density, $\mathrm{kg} / \mathrm{m}^{3}$

$\sigma \quad$ relative supersaturation, dimensionless

$\sigma_{l} \quad$ interfacial supersaturation, dimensionless

\section{Acknowledgment}

The authors gratefully acknowledge the financial support provided by the National Science Council of Republic of China (Taiwan).

\section{References}

ASTM Designation D513, 1974, p. 416.

Budz, J., Karpinski, P.H., Nuruc, Z., 1984. Influence of hydrodynamics on crystal growth and dissolution in a fluidized bed. A.I.Ch.E. Journal 30, $710-717$.

Butler, J.N., 1964. Ionic Equilibrium. Addison-Wesley, Reading MA, p. 284.

Dirken, P., Barrs, E., Graveland, A., Woensdregt, C.F., 1990. On the crystallization of calcite $\left(\mathrm{CaCO}_{3}\right)$ during the softening process of drinking water in a pellet reactor with fluidized beds of quartz, garnet and calcite seeds. In: Mersmann, A. (Ed.), Industrial Crystallization 90, GarmischPartenkrichen, FRG, p. 95.

Fukui, K., Nakajima, M., 1985. Diffusion processes near the crystal surface in growth and dissolution. Journal of Chemical Engineering (Japan) 18, 27-32.

Mullin, J.W., 1993. Crystallization. Butterworth-Heinemann, Oxford, pp. 203-217.

Nancollas, G.H., 1966. Interaction in Electrolyte Solutions. Elsevier, Amsterdam, p. 80.

Nielsen, A.E., Toft, J.M., 1984. Electrolyte crystal growth kinetics. Journal of Crystal Growth 67, 278-288.

Phillips, V.R., Epstein, N., 1974. Growth of nickel sulfate in a laboratoryscale fluidized-bed crystallizer. A.I.Ch.E. Journal 20, 678-687.

Reed, J.S., 1989. Principles of Ceramic Processing. Wiley, Singapore, p. 134.

Stubicar, N., Scrbak, M., Stubicar, M., 1990. Crystal growth of lead fluoride using the constant composition method, II. The effect of $\mathrm{Pb} / \mathrm{F}$ activity ratio on the kinetics of crystal growth. Journal of Crystal Growth 100, 261-267.

Stubicar, N., Markovic, B., Tonejc, A., Stubicar, M., 1993. Crystal growth of lead fluoride phases using the constant composition method, III. Effect of $\mathrm{pH}$ and ionic strength. Journal of Crystal Growth 130, 300-304.

Tai, C.Y., 1997. Crystallization kinetics revealed from experimental data analyzed by the two-step growth model. Journal of Chemical Engineering (Japan) 30, 373-381.

Tai, C.Y., Pan, R.-K., 1985. Growth kinetics of copper sulfate pentahydrate crystal in pure and impure systems. Journal of Chinese Institute of Chemical Engineers 16, 379-387. 
Tai, C.Y., Chen, C.-Y., Wu, J.-F., 1987. Crystal dissolution and growth in a lean fluidized-bed crystallizer. Chemical Engineering Communication 56, 329-340.

Tai, C.Y., Chen, P.-C., Shih, S.-M., 1993. Size-dependent growth and contact nucleation of calcite crystals. A.I.Ch.E. Journal 39, 1472-1482.

Tai, C.Y., Chien, W.-C., Chen, C.-Y., 1999. Crystal growth kinetics of calcite in a dense fluidized-bed crystallizer. A.I.Ch.E. Journal 45, 1605-1614.
Tai, C.Y., Lu, C.H., Wu, C.K., 2005. Crystal growth rate of calcite in a constant-composition environment. Journal of Chinese Institute of Chemical Engineers 36, 443-450.

Van Dijk, J.C., Wilms, D.A., 1991. Water treatment without waste material-Fundamentals and state of the art of pellet softening. Journal of Water SRT-Aqua 40, 263-280. 